\title{
Liquid-crystal enabled electrophoresis: Scenarios for driving and reconfigurable assembling of colloids
}

\author{
Sergi Hernàndez-Navarro ${ }^{1,3}$, Pietro Tierno ${ }^{2,3}$, Jordi Ignés-Mullol ${ }^{1,3}$, and Francesc \\ Sagués 1,3, a \\ 1 Departament de Química Física, Universitat de Barcelona, Martí i Franquès 1, 08028 \\ Barcelona, Spain. \\ 2 Department of Structure and Constituents of Matter, Universitat de Barcelona, Diagonal \\ 647, 08028 Barcelona, Spain. \\ ${ }^{3}$ Institute of Nanoscience and Nanotechnology, $\mathrm{IN}^{2} \mathrm{UB}$, University of Barcelona.
}

\begin{abstract}
We demonstrate several examples of driving and steering of colloids when dispersed in nematic liquid crystals. The driving mechanism is based on the principle of nonlinear electrophoresis which is mediated by the asymmetry in the structure of the defects that the inclusions generate in the host elastic matrix. The steering mechanism originates in the photoactivation of the anchoring conditions of the nematic liquid crystal on one of the enclosing plates. As experimental realizations we first review a scenario of water microdroplets being phoretically transported for cargo release and chemical reaction. Steering is illustrated in terms of the reconfigurable assembly of colloidal particles, either in the form of asters or rotating-mills, commanded by predesigned patterns of illumination.
\end{abstract}

\section{Liquid crystals as dispersing media for colloids}

One of the most distinctive properties of soft matter systems is that they are extremely labile, and as such they may be readily addressable by means of appropriately chosen external fields. However, this capability is useless if one does not guarantee a sufficient control over the elicited responses, particularly when these responses are prone to be highly degenerated. This is particularly true when referring to two of the most studied categories of soft materials: liquid crystals and colloids. In particular, interest has increased enormously in recent years to bridge the potentialities of both systems through the use of liquid crystals as dispersing media for solid or liquid colloidal inclusions. Such composite systems, where we benefit of the discrete nature of the dispersed components and, at the same time, of the orientational properties of the liquid crystal medium, constitute nowadays familiar mixtures under intense scrutiny to unveil new fundamental concepts and original applications [1-3]. In particular, the capacity of the elastic and anisotropic host matrix to mediate interactions be-

\footnotetext{
${ }^{a}$ e-mail: f.sagues@ub.edu
} 
tween suspended inclusions was already discovered many years ago [4], and has been profusely used since then [5-14].

In contrast to research on liquid crystal dispersions of sub-micron or nano-scale particles $[15,16]$, we concentrate in what follows on systems that contain colloidal inclusions at the micrometer scale, which enable a real discrete control and observation of the dispersed components. It is also worth emphasizing at this point that, in spite of very recently investigated dispersions prepared with lyotropic (i.e. water-based) liquid crystals $[17,18]$, all what we are going to review in this contribution refers to thermotropic oily materials in their nematic phases [19].

Nematic liquid crystals (NLCs) are complex fluids mostly used in display technologies. They are characterized by rod shaped organic molecules which tend to align their long axis along one direction, called the director field. The behavior of colloids dispersed in NLCs is largely determined by considering the state of the elastic LC matrix around the dispersed inclusion. In this respect, the most important characteristics is the anchoring conditions of the liquid crystal at the surface of the colloid. The simplest case is that of a spherical solid particle, around which the nematic material may adopt either a tangential (parallel) or a homeotropic (perpendicular) arrangement, depending on the particular system and surface preparation. In either case, placing a colloidal unit into an otherwise aligned nematic phase leads to topological frustration and to the appearance of defects (i.e. singularities in the distribution of the director field), that are tightly bound to the inclusion. For homeotropic anchoring, two main types of defects may arise: a point-defect known as a hyperbolic hedgehog or a disclination loop extending as an equatorial band and known as Saturn-ring [20-22]. For tangential anchoring, the defect structure is that of a double-boojum [20]. Schematic representations of these defect configurations are presented in Fig. 1. Of singular importance on what follows is the elucidation of the symmetries of these defects. In this respect, Poulin et al., already pointed out in their seminal paper [4] the convenience to map the colloidal-induced elastic distortion of the far field distribution of the nematic director onto an electrostatic context. According to this analogy, a (ideal) spherical colloid with its corresponding hedgehog defect acts as an electrostatic dipole, whereas the structures of Saturn-rings and double-boojums are better understood as giving rise to quadrupolar interactions.

\section{From non-linear electrophoresis to liquid-crystal enabled electrophoresis}

Another more recently investigated feature of nematic colloids, this term used as a short denomination for colloidal dispersions in a continuous nematic phase, is the possibility to observe non-trivial manifestations of phoretic driving of the dispersed inclusions under the application of electric fields. Indeed, what makes this possibility particularly striking is that, contrarily to normal phoresis under steady fields, the motion is here induced by oscillating electric fields. This confers to the phenomenon a distinctive non-linear character that justifies the use of the abbreviated denomination non-linear electrophoresis [23]. An alternative way to qualify this electrically-driven transport, more appropriate to its physical origin, is known as induced-charge electroosmosis [24]. Focussing on liquid crystal based systems, we will employ on what follows the composed form liquid-crystal enabled electrophoresis LCEEP, as was recently coined by Lavrentovich et al. [25].

For normal electrically induced phoresis, particles are transported under uniform DC fields with a velocity that depends linearly on the applied field. Obviously this means that oscillating fields which average to zero over a period would cause a zero displacement. However the possibility to use alternate rather than steady driving 
a

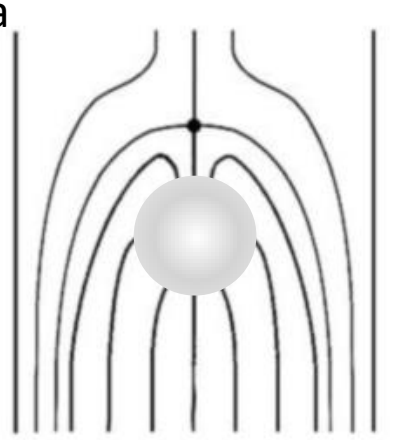

b

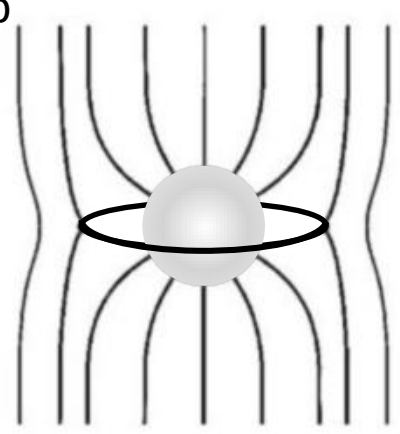

C

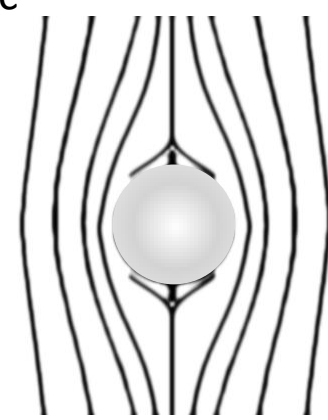

Fig. 1. Different defect structures for inclusions dispersed in nematic liquid crystals. a) Hedgehog configuration; b) Saturn-ring disclination; c) Double-boojum structure. The first arrangement has a dipolar symmetry, whereas the other two feature distortions of quadrupolar nature.

modes has the advantatge that concurrent electrochemical processes, that take place in the normally aqueous medium employed in electrophoretic cells, are avoided. In fact, nonlinear versions of electrophoresis were first reported for isotropic fluids more than twenty years ago [26], as mentioned in the recent review [25], and were exhaustively analyzed in some theoretical papers by Bazant and Squires [24]. In this latter study, the main conclusion is that a nonlinear electro-osmotic slip occurs when an applied field acts on the ionic charge it induces around a polarizable surface. Notice from this very first statement that nonlinear electrokinetic phenomena apply to charged and non-charged inclusions as well, in striking contrast with normal electrophoresis. As a matter of fact, the possibility to further break the symmetries of the resulting flow and obtain direct motion for Janus-like particles (metallo-dielectric) was first published by Velev et al. [27].

In the context of liquid crystals, the breaking of the fore-aft symmetry that guarantees direct transport may be achieved even for perfectly spherical particle bearing in mind our above considerations relative to the symmetries of the nematics director field around the inclusions. This is quite evident for defects with dipolar symmetry, but also applies to distorted quadrupolar symmetries. The latter situation would occur, for instance, in the case of a parallel anchoring of the liquid crystal material wrapping around non-perfectly symmetric, i.e., anisometric, particles. Both possibilities have been largely exploited in our recent experiments that will be reviewed in the second part of this contribution. Notice finally that a quadratic dependence of the phoretic velocity with respect to the applied field has another striking implication: the dependence of the velocity on the applied electric field, both vectorial quantities, must be indeed of a tensorial nature. This, in turn, permits that the direction of motion and that of the electric field are non-necessarily parallel [23] . A general overview on the different possibilities involved in the transport of particles in liquid crystals is provided in [25].

\section{Phoresis of nematic colloids: an active matter-based perspective and applications in materials science}

Active matter is a presently very celebrated keyword under which we recognize many forms of elementary living matter, altogether with remarkable non-living soft matter analogs [28]. A defining feature commonly exhibited by the units composing these 
discrete systems is their ability for autonomous or driven motion. More precisely, we should properly reserve the term active for the first kind of (self-driven) behavior and employ the term actuated to refer to the second situation, which is in fact what applies to our electrophoretic colloids dispersed in a liquid crystal material. Under whatever acception, much attention has been devoted to elucidate the mechanisms behind complex multiparticle phenomena that self-organize into characteristic patterns with long range order, either of static or dynamic nature. In this respect, terms like bands, asters, vortices are very much familiar to practitioners in this field. Diverse aspects of this topic are examined in this Proceedings volume while a comprehensive update is provided in the review by Marchetti et al. [28]). Although experimental realizations are becoming more and more impressive particularly when restricting to a bio-inspired context, see [29-31] for striking observations of active self-organization in a biophysical context, we feel that theoretical and numerical work is leading the activity in the field. We thus claim for the need to provide the interested community with experimentally robust realizations of new scenarios of self-organized patterns in soft-matter systems, either active or driven. More important, as it is going to be much emphasized on the last part of this contribution, is the further need to gain control on the assembly process and to demonstrate the eventual possibility to reconfigure these aggregates at will.

We would like to mention another completely different and more materials-based perspective from where our experimental research on phoretic colloids could be addressed. The assembly and transport of colloidal entities, such as particles, droplets or microorganisms have direct applications in fields such as photonics [32], lab-ona-chip technologies [33] and biomedicine [34]. During this last decade the focus on self-assembling started to shift from equilibrium characteristics to dynamic aspects [35], seeking enhanced functionalities of the resulting materials [36]. Optical trapping techniques are often employed to achieve direct control over the placement of colloidal inclusions, and holographic tweezers allow to extend such control to a few hundreds of particles [37], although this technique is limited by the field of view of the optical system. Going beyond earlier work on physically and chemically activated colloids [38-40], a recent realization explores the idea of phoretic colloids driven by osmotic pressure imbalances [41]. These results have opened new possibilities for reconfigurable self-assembly, enabling the massive transport of inclusions [42], although the precise and selective control on such dynamical patterns lacked demonstration.

\section{AC electrophoresis of microdroplets in liquid crystals: transport and reaction}

The second part of this contribution is prepared to briefly summarize our recent research on the phoresis of inclusions dispersed in nematic liquid crystals. It is itself divided into two sections. The first one is devoted to the transport of (water) microdroplets, while we reserve the second part to present a new development that permits the reconfigurable assembly of colloidal particles into clusters (swarms).

Water-based microemulsions prepared in oil phases are of fundamental importance in chemical and analytical sciences, due to the possibility of encapsulating and delivering chemical compounds otherwise immiscible in the dispersion medium. The possibility to remotely transport microdroplets is thus singularly appealing. Electrically induced phoresis is undoubtedly the first considered alternative, and particularly its non-linear version based on the use of oscillating electric fields offer great advantages as mentioned previously in Sect. 2 .

Our experimental system, thoroughly described in Hernàndez-Navarro et al. [44], permits to transport water microdroplets dispersed in a nematic liquid crystal with 


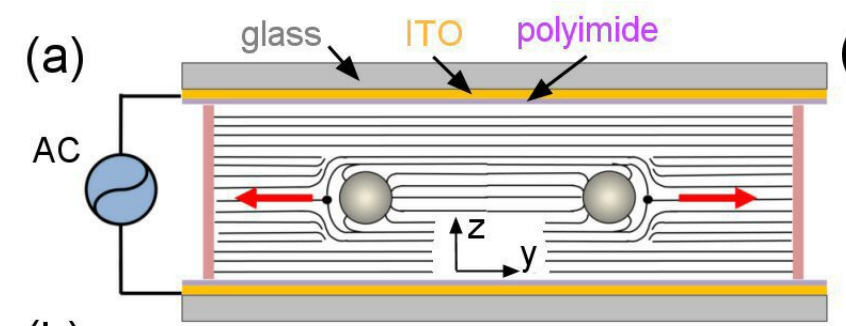

(b)

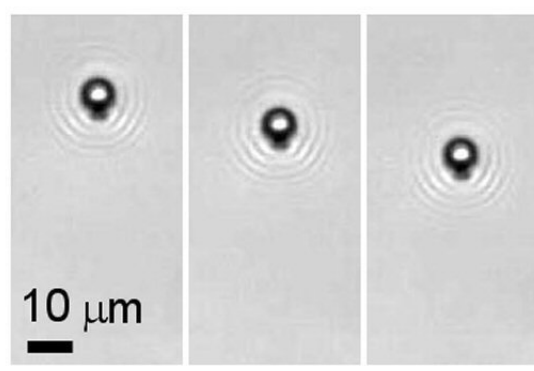

(c)
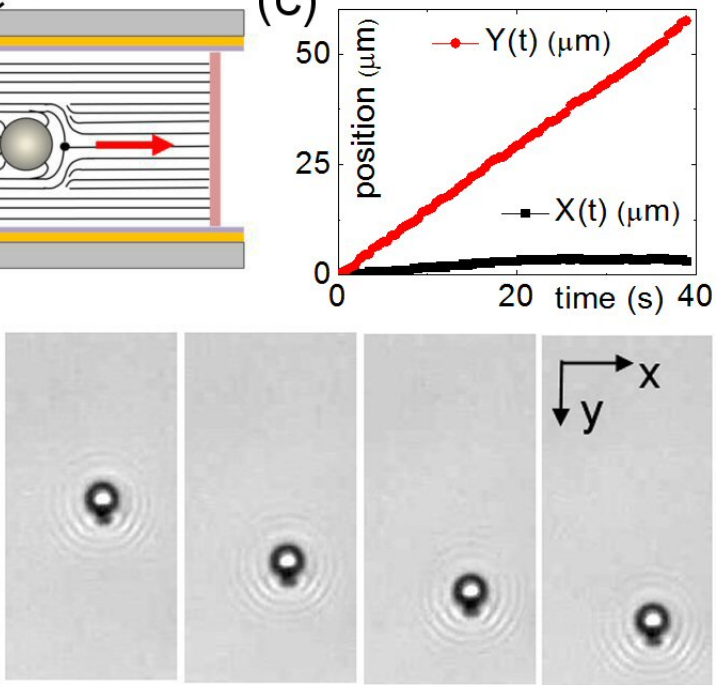

Fig. 2. (a) Schematics of the experimental cell with water microdroplets dispersed in a nematic liquid crystal contained between two transparent glass electrodes functionalized to favor planar anchoring. The water droplets are propelled by the mechanism of LCEEP (see text). (b) Optical micrographs taken every $4.5 \mathrm{~s}$ of a water droplet having a $6.5 \mu \mathrm{m}$ diameter and moved at a speed of $1.7 \mu \mathrm{m} \mathrm{s}^{-1}$ by an AC field of amplitude $\mathrm{E}=0.7 \mathrm{~V} \mathrm{\mu \textrm {m } ^ { - 1 }}$ and frequency, $f=10 \mathrm{~Hz}$. (c) Positions ( $\mathrm{x}, \mathrm{y}$ ) versus time of the droplet shown in panel (b). Image reproduced with permission from [44]. Copyright Royal Society of Chemistry 2013.

negative dielectric anisotropy, i.e. it allows to observe phoretic motion perpendicular to the applied electric field. Additionally, we decouple in this way AC electrophoresis from any residual linear DC contribution. As reported in the reference mentioned, we were able to demonstrate not only droplet motion but to show in addition that these droplets can be used as microreactors to transport sub-micrometric particles or to mix tiny volumes of chemicals.

The used cell was composed of two $0.7 \mathrm{~mm}$ thich microscope slides of size $15 \times 25$ $\mathrm{mm}^{2}$ coated with a layer of indium-tin oxide (ITO). The two slides were cleaned, dried and further chemically treated to obtain a planar alignment of the liquid crystal on the bounding plates. These were separated by a spacer of nominal thickness 23 microns and glued together with the ITO layers facing inwards. Dispersions of aqueous microdroplets (from 1 to 20 micron diameter sizes) were prepared by vortex agitation using MLC-7029 as a nematic phase, using as stabilizing agent sodium dodecyl sulphate (SDS). This protocol guarantees a homeotropic alignment of the nematics on the droplets surface. The experimental cells were filled by capillarity. Sinusoidal electric fields were applied by using a function generator, within a range of 0 to 30 volts peak-to-peak, while the applied range of frequencies varied from 0 to $100 \mathrm{~Hz}$. Experimental observations were performed with an optical microscope, and images were captured with a recording camera controlled with the appropriate software AVT SmartView 1.10.2, and further treated using software packages ImageJ and IgorPro. For more details see the original paper [44].

Figure 2(a) shows the experimental cell. In the scenarios we are reviewing here we found that the defects were predominantly of the hedgehog type, while only a small fraction of inclusions (less than 1\%) featured Saturn-ring defects. Motion was always observed in the direction towards the point defect as clearly observed if Fig. 2(b). The 
(a)
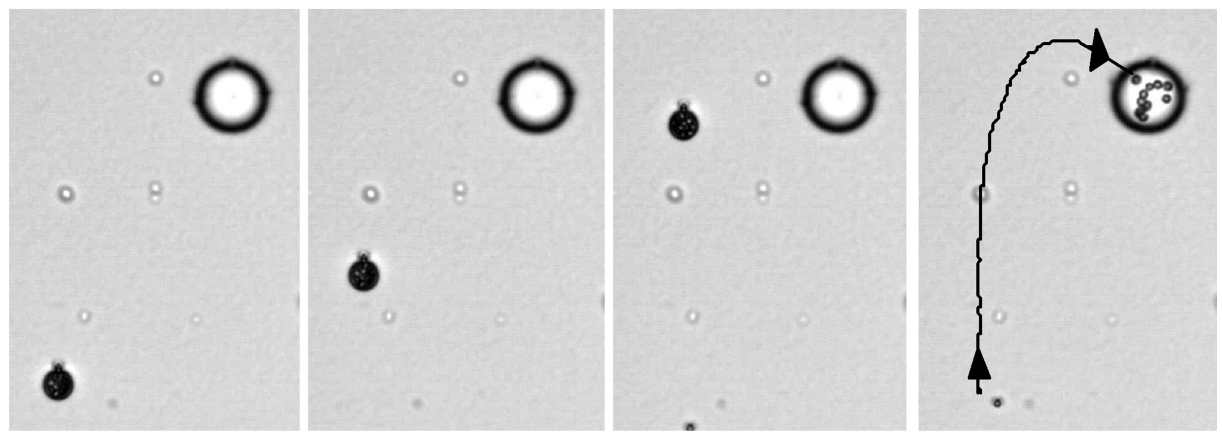

(b)
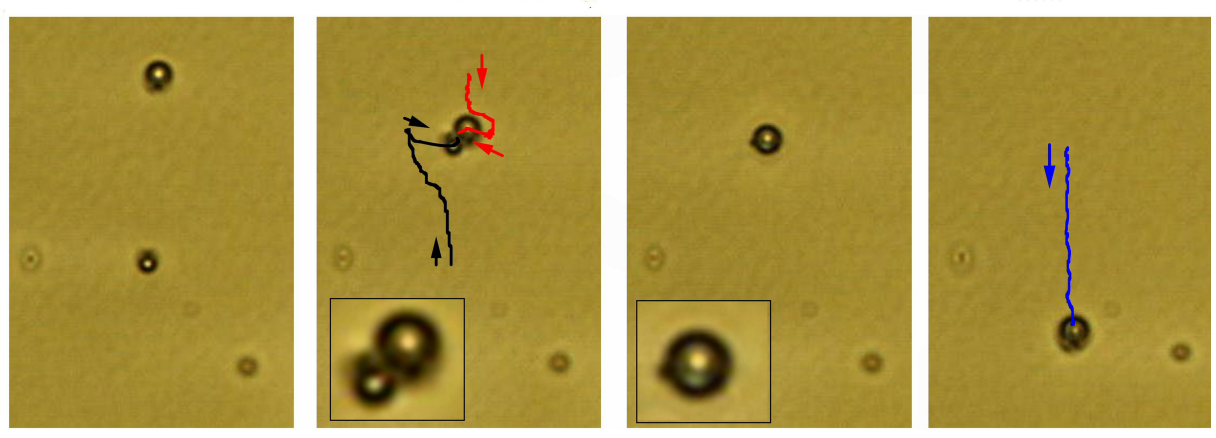

Fig. 3. (a) Sequence of images showing the coalescence of a microdroplet of $7.3 \mu \mathrm{m}$ diameter filled with 11 smaller polystyrene particles with a large droplet of diameter $18 \mu \mathrm{m}$ bearing a Saturn-ring defect $\left(\mathrm{E}=0.70 \mathrm{~V} \mu \mathrm{m}^{-1}, \mathrm{f}=10 \mathrm{~Hz}\right)$. Time interval between images is 17.2 s. (b) Microscope images showing two water microdroplets with diameters 2.7 and $3.7 \mu \mathrm{m}$ driven in opposite directions by an AC field of the same characteristics. Droplets contain separated reactants, respectively potassium ferrocyanide $(0.2 \mathrm{M})$ and ferric ions $(0.3 \mathrm{M})$. The microdroplets approach and coalesce into a larger one forming Prussian blue as a precipitate. Image reproduced with permission from in [44]. Copyright Royal Society of Chemistry 2013.

measured velocities are typically on the range of a few microns per second, featuring the expected quadratic dependence on the amplitude of the electric field and a peak at intermediate frequencies (tens of Hertz).

To further demonstrate the possibilities of this phoretic motion, a localized cargo release operation is shown in Fig. 3 (a). A microdroplet loaded with polystyrene particles is driven towards a larger droplet bearing a Saturn-ring defect, and thus stays at rest under the applied electric field. The larger droplet elastically attracts the small one, and the colloidal cargo is released by droplet coalescence. In Fig. 3 (b), two droplets loaded with specific reactants and featuring antiparallel configurations of their respective dipolar defect configuration attract each other, and their coalescence triggers a chemical precipitation reaction (see original paper [44] for further details).

\section{Reconfigurable swarming}

Reconfigurability is a genuine property of living active matter. As laboratory-model systems, collections of driven colloids have a potential for large-scale addressability. Here we review our very recent research that indeed demonstrate real-time reconfigurable clustering of phoretic colloids dispersed in thin nematic layers in response to illumination patterns. Swarms of particles are shown to reversible organize in submillimeter ensembles that can be individually or collectively addressed to change their 

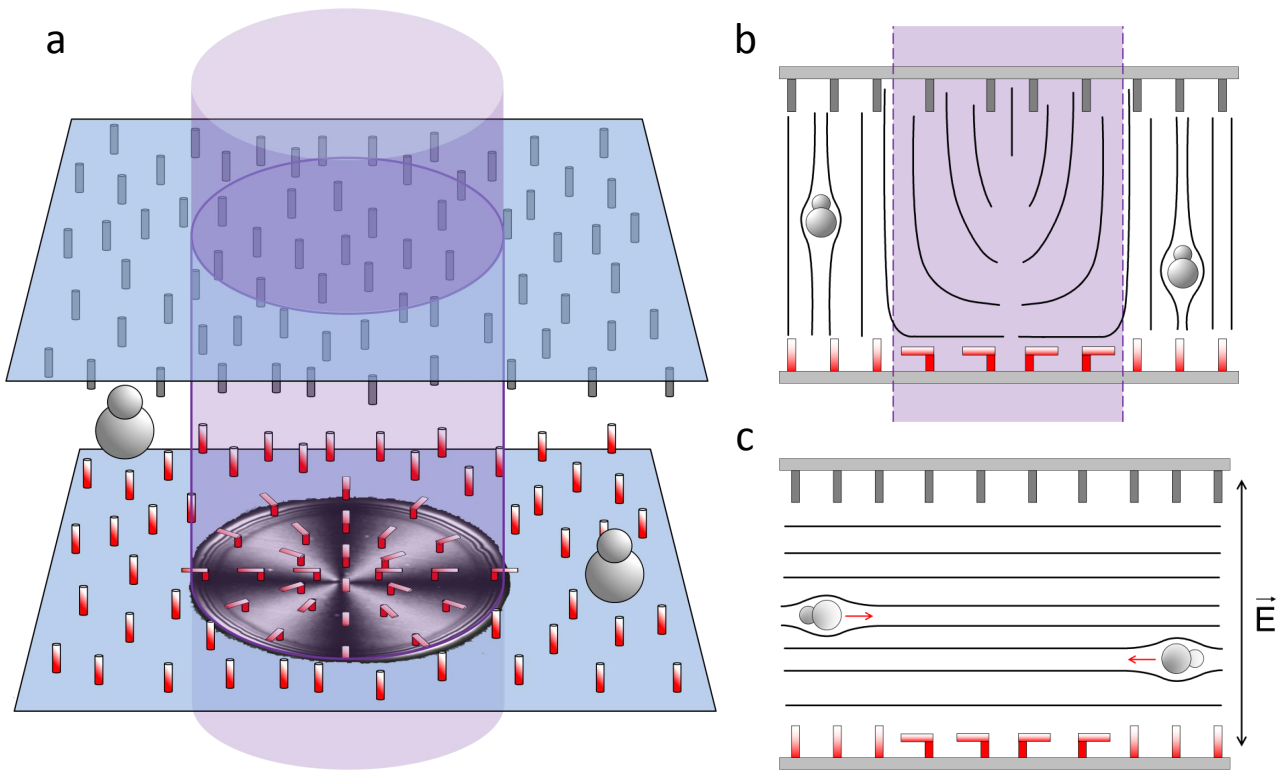

Fig. 4. (a)Scheme of the experimental cell as a radial pattern is imprinted using UV light $(365 \mathrm{~nm})$. The bottom plate is functionalized with an azosilane derivative, and the upper plate presents homeotropic anchoring. (b) Scheme of a transversal cut of the experimental cell. (c) Scheme of a transversal cut of the experimental cell, upon application of a sinusoidal electric field. Red arrows indicate the dominant direction of motion of the anisometric particles.

position or dynamic behavior. The strategy we follow permits to separate particle steering, achieved through photoelastic modulation of the host nematic, and particle driving realized through LCEEP. A complete account of these experiments can be found in Hernàndez-Navarro et al. [45].

We used the same nematic liquid crystal as in the previously reported experiments, thus favoring motion of the dispersed colloids perpendicular to the applied electric field. However, the chemical treatments of the confining plates was different in this case and, in fact they were specifically aimed at achieving the purposed control on the assembly of the dispersed particles. One of the plates was chemically functionalized with an azosilane photosensitive self-assembled monolayer that allows to alternate between perpendicular (homeotropic) and tangential (planar) anchoring of the nematic. The counter plate was coated with a polyimide compound to guarantee strong and permanent perpendicular contact. Without any external influence, such boundary conditions lead to a uniform homeotropic texture within the whole cell. By irradiating with UV (365 nm) light from an incoherent source the azosylane is forced to adopt the cis configuration, and consequent planar boundary condition, easily reverted to trans form, and corresponding homeotropic anchoring, using blue $(455 \mathrm{~nm})$ illumination.

As colloidal inclusions we used pear-shaped microparticles made of polystyrene, a material that promotes planar orientation of the NLC on the particle surface. The chosen particle shape guaranteed a distorted quadrupolar symmetry arising from the defect distribution around the particles. In the absence of irradiation or electric field, particles align perpendicular to the cell plates, following the uniform director field. Shinning the cell for a few seconds with a spot of UV light forces the NLC in contact with the azosylane-treated surface to transit to a planar configuration by locally adopting a local splayed (radially-spread) texture emanating from a central defect. 

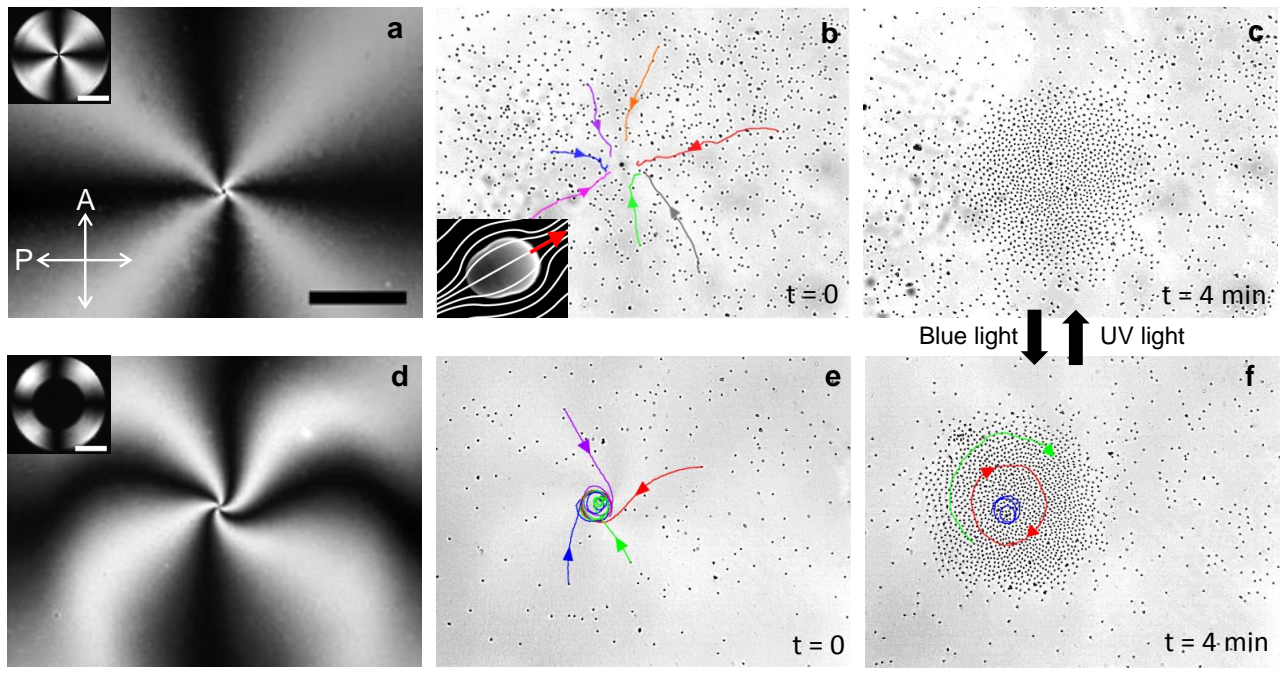

Fig. 5. Top (a-c) row of images illustrates the formation of a colloidal aster. Bottom (d-f) row corresponds to the assembly of a rotating mill-like cluster. (a) and (d) are images between cross polarizers of the imprinted texture leading to a cross (a) or a spiral (d) attracting pole. The inset shows the planar photoaligned circle (inset (a)) or corona (inset (d)) prior to the application of the electric field. The applied electric field in both cases has an amplitude of $0.87 \mathrm{~V} \mu \mathrm{m}^{-1}$ and a frequency of $10 \mathrm{~Hz}$. Trajectories followed by the several particles are superimposed to the images. Inset in (b) shows a S.E.M. image of a single pear-shaped particle $(3 \mu \mathrm{m} \times 4 \mu \mathrm{m})$. The two cluster modes can be interconverted by suitable irradiation protocols as indicated. The scale bars are $200 \mu \mathrm{m}$ for all images and $500 \mu \mathrm{m}$ for the insets. Image adapted with permission from [45]. Copyright Wiley 2014.

Application of an external AC field makes the bulk NLC to adopt the splay configuration that now extends for several millimeters, well beyond the area of the irradiated spot, thanks to the homeotropic anchoring degeneracy relative to any planar direction. This configuration is stable for days under AC field, well past the half-life for thermal relaxation of the azosylane film, which is about 30 minutes. The region with radial alignment will be the basin of attraction for dispersed particles, which tumble instantaneously following the NLC director so that their long axis lays, on average, parallel to the cell plates. Simultaneously the LCEEP sets the particles into motion at a constant speed. All particles moved following the local NLC director, with roughly half of them being attracted by the photoinduced radial defect and the rest being repelled from it. This is direct consequence of the random tumbling of the particle following the reorientation of the liquid crystal matrix. See Fig. 4 for a combined schematics of the different aspects of the optical and electrical forcing of the cell.

The course of an standard experiment triggered the accumulation of particles, as they follow the nematic field lines. A growing aster-like cluster was typically observed after the progressive arrival and jamming of the particles at high density assemblies, see Fig. 5 (panels a, b, c). We could easily switch to a dynamic structure, a rotating mill-like cluster, by profiting from the elastic properties of the nematic material (smaller value of the bend elastic constant with respect to the splay counterpart) and the fact that the particles are totally slave to the director. The experimental protocol proceeds by erasing the central region of an imprinted UV area with a smaller spot of blue light, prior to the application of the electric field. Following the inverse isomerization to the trans form of the azosylane anchoring derivative, the NLC director features a homeotropic configuration both outside and inside a corona with planar 
alignment. Upon application of the AC field, the NLC director adopts a degenerate planar alignment both inside and outside the ring. The energy cost of the large splay distortion inside the ring prompts the director to adopt a spiral bend-splay texture and director field lines conform to a spiral-like geometry. As a consequence particles assemble into a rotating mill structure, preceding around the central defect with a constant linear velocity, see Fig. 5 (panels d, e, f). Both assembly modes can be reversibly interconverted in real time via the photoativation control just described (see original reference [45]).

The reversibility and quick response of the photoalignment layer enables straightforward cluster addressability. A preformed aggregate of arbitrary size, either aster or vortex-like, can be relocated to a pre-designed place anywhere within the experimental cell with minimum dismantlement of the cluster structure by changing the location of the UV spot. An example of this process is shown in 6. After blocking the LCEEP mechanism by increasing the field frequency above $50 \mathrm{~Hz}$, the center of attraction is translated $600 \mu \mathrm{m}$. Once LCEEP is reactivated, the swarm of particles moves towards the new position developing a leading edge around which the particles assemble. The space-time plot illustrates the resulting collective behavior. Alternatively, by the same principle one can imprint predesigned arbitrary paths connecting distant locations inside the cell or draw circuits with complex topologies as a simple way to accumulate colloidal swarms in the irradiated area and further entrain them collectively

We thank Patrick Oswald for the polyimide compound. We acknowledge financial support by MICINN (FIS2010-21924C02, FIS2011-15948-E) and DURSI (2009 SGR 1055). S.H.-N. acknowledges support through an FPU Fellowship (AP2009-0974). P.T. further acknowledges support from the ERC through the starting grant DynaMO (335040) and from the Ramon y Cajal program (RYC-2011-07605).

\section{References}

1. A. Agarwal et al., Small 9, (2013) 2785

2. B. Senyuk et al., Nature 493, (2013) 200

3. T. A. Wood et al., Science 334, (2011) 79

4. P. Poulin et al., Science 275, (1997) 1770

5. J. C. Loudet et al., Nature 407, (2000) 6111

6. J. Yamamoto et al., Nature 409, (2001) 322

7. C. Lapointe et al., Science 303, (2004) 652

8. M. Yada et al.,Phys. Rev. Lett. 92, (2004) 185501

9. I. Musevic et al., Science 313, (2006) 954

10. O. P. Pishnyak et al., Phys. Rev. Lett. 99, (2007) 127802

11. C. P. Lapointe et al., Science 326, (2009) 1083

12. G. M. Koening et al., Proc. Natl. Acad. Sci. USA 107, (2010) 3998

13. U. Tkalec et al., Science 333, (2011) 62

14. R. P. Trivedi et al., Proc. Natl. Acad. Sci. USA 109, (2012) 4744

15. H. Qi et al., Adv. Funct. Mat. 18, (2008) 212

16. S. Acharya et al., Adv. Mat. 21, (2009) 989

17. S. Zhou et al., Proc. Natl. Acad. Sci. USA 111, (2014) 1265

18. P. C. Mushenheim et al., Soft Matter 10, (2014) 88

19. P. Oswald et al., Les Cristaux Liquides, Gordon and Breach Sci. Pub., Paris 2000

20. P. Poulin et al., Phys. Rev. E 57, (1998) 626

21. T. C. Lubensky et al., Phys. Rev. E 57, (1998) 610

22. Y. Gu et al., Phys. Rev. Lett. 85, (2000) 4719 

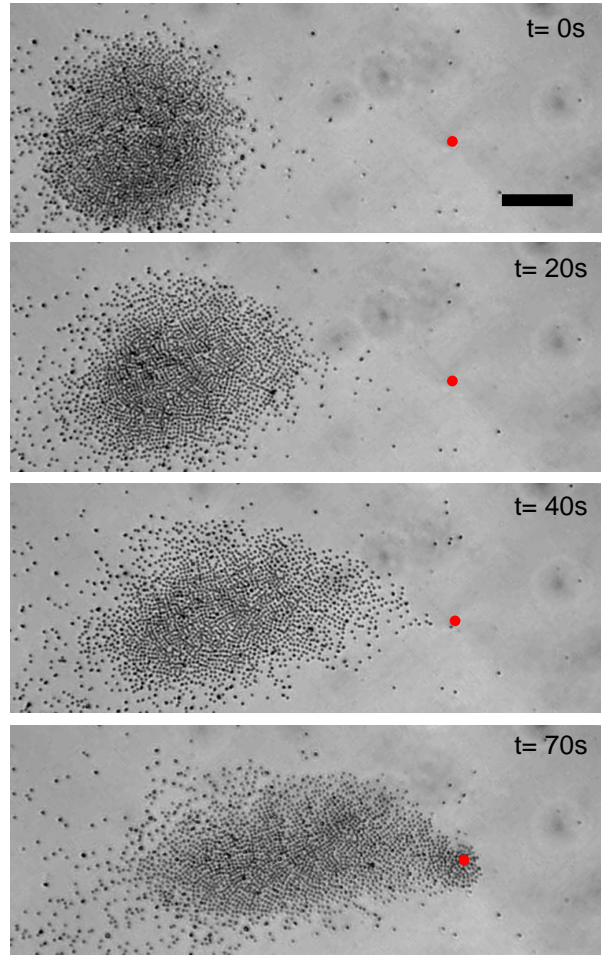
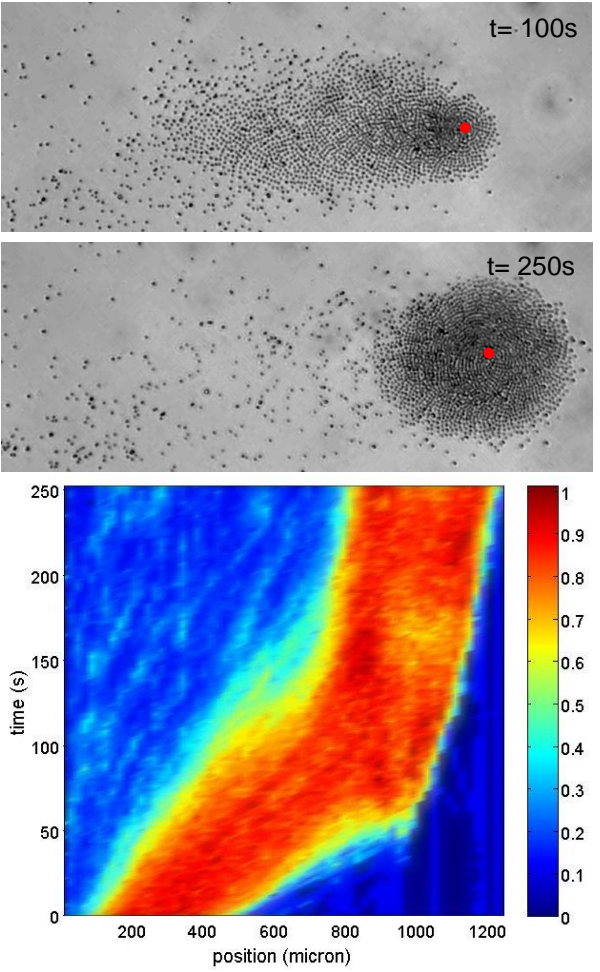

Fig. 6. Image sequence of a particle swarm traveling across the LC cell due to in situ reconfiguration of the NLC field. The photoaligned spot initially centered with the cluster is moved $600 \mu \mathrm{m}$ to the right, as indicated by the red spot. The applied sinusoidal electric field has an amplitude of $0.74 \mathrm{~V} \mu \mathrm{m}^{-1}$ and a frequency of $10 \mathrm{~Hz}$. The chart is a space-time plot visualizing the swarm dynamics during the experiment. The normalized average particle density across different positions is color encoded, with dark blue corresponding to particle depleted and dark red to particle rich regions. Scale bar marks $150 \mu \mathrm{m}$.

23. O. D. Lavrentovich et al., Nature 467, (2010) 947

24. T. M. Squires et al., J. Fluid. Mech, 509, (2004) 217

25. O. D. Lavrentovich et al., Soft Matter 10, (2014) 1264

26. V. A. Murtsokvin et al., Colloid J. 52, (1990) 933

27. S. Gangwal et al., Phys. Rev. Lett. 100, (2008) 058302

28. M. C. Marchetti et al., Rev. Mod. Phys. 85, (2013) 1143

29. V. Schaller et al., Nature 467, (2010) 73

30. Y. Sumino et al., Nature 483, (2012) 448

31. T. Sanchez et al., Nature 491, (2012) 431

32. A Yethiraj et al.,Adv. Mat. 16, (2004) 596

33. A. Terray et al., Science 296, (2002) 1841

34. E. C. Dreaden et al., Chem. Soc. Rev. 41, (2012) 2740

35. G. M. Whitesides et al., Science 295, (2002) 2418

36. N. I. Zheludev et al., Nat. Mat. 11, (2012) 917

37. D. G. Grier, Nature 424, (2003) 810

38. W. F. Paxton et al., J. Am. Chem. Soc. 126, (2004) 13424

39. J. R. Howse et al., Phys. Rev. Lett. 99, (2007) 048102

40. I. Buttinoni et al., Phys. Rev. Lett. 110, (2013) 238301

41. J. Palacci et al., Science 339, (2013) 936

42. A. Bricard et al., Nature 503, (2013) 95

43. J. Guzowski et al., Soft Matter 8, (2012) 7269 
44. S. Hernàndez-Navarro et al., Soft Matter 9, (2013) 7999

45. S. Hernàndez-Navarro et al., Angew. Chem. Int. Ed. 53, (2014) 10696 